\title{
Divergent Mathematical Treatments in Utility Theory
}

\author{
Davide Rizza ${ }^{1}$
}

Received: 19 January 2015/Accepted: 24 November 2015/Published online: 14 December 2015

(C) The Author(s) 2015. This article is published with open access at Springerlink.com

\begin{abstract}
In this paper I study how divergent mathematical treatments affect mathematical modelling, with a special focus on utility theory. In particular I examine recent work on the ranking of information states and the discounting of future utilities, in order to show how, by replacing the standard analytical treatment of the models involved with one based on the framework of Nonstandard Analysis, diametrically opposite results are obtained. In both cases, the choice between the standard and nonstandard treatment amounts to a selection of set-theoretical parameters that cannot be made on purely empirical grounds. The analysis of this phenomenon gives rise to a simple logical account of the relativity of impossibility theorems in economic theory, which concludes the paper.
\end{abstract}

\section{Model Theory and Scientific Models}

In a 1960 paper, Patrick Suppes claimed that:

[...] in the exact statement of the theory or in the exact analysis of data the notion of model in the sense of logicians provides the appropriate intellectual tool for making the analysis both precise and clear. (Suppes 1960: 295)

This claim was defended against the background thesis that the meaning of the concept of model is the same in mathematics and the empirical sciences (Suppes 1960: 289). Suppes' view of models is too restrictive in two distinct ways: one of these has become clear through the recent literature on modelling, whereas the other has been neglected and provides the main motivation for the discussion presented in

Davide Rizza

d.rizza@uea.ac.uk

1 School of Politics, Philosophy, Language and Communication Studies, University of East Anglia, Norwich NR4 7TJ, UK 
this paper. The first sense in which Suppes' view is too restrictive is that it wishes to assimilate models tout court to mathematical models. Later developments in philosophy of science have moved away from this perspective, while acknowledging the significance of Suppes' proposal. For instance, the several contributions included in Morgan and Morrison (1999), a turning point in the philosophy of scientific modelling, work with a notion of model that is much more wide-ranging than the one proposed by Suppes. This is perhaps most clearly stated in Adrienne van den Bogaard's contribution to the collection, when she writes:

Arguments about the model as a theory, or a method, or a distortion of reality, all focus on the model as a scientific object and how it functions in science. Without denying this dimension of the model at all, this paper wants to broaden the perspective by claiming that the model is also a social and political device. The model will be understood as a practice connecting data, index numbers, national accounts, equations, institutes, trained personnel, laws, and policy-making. (van den Bogaard 1999: 283)

It is clear that, if one is ready to accept the qualification of models for methods, distortions of reality and social devices, Suppes' more stringent semantic qualification must appear to impose very narrow, perhaps unrealistic, constraints on the study of modelling practices. It does not follow that Suppes' appeal to notions and techniques from mathematical logic should be deemed irrelevant to the study of modelling practices in general. In this paper, I seek to defend the opposite point of view by applying a model-theoretic approach to the study of mathematical modelling within utility theory. While doing so, I depart from Suppes' original aims, which presuppose, in my opinion, too strict a delimitation of the ways in which model-theoretic considerations may support philosophical investigations of scientific models. The quotation opening this section spells out the delimitation in question by restricting the mobilisation of set-theoretical semantics to the purposes of formulating scientific theories (typically as classes of models defined by a settheoretical predicate, an approach whose abstract development has been presented in Da Costa and Chuaqui 1988) or carrying out an exact analysis of data (e.g. by the embedding of a data structure into a representing structure, a strategy adopted in Da Costa and French 2003). These investigations are of clear philosophical interest, but they rely more on the set-theoretical representation of structures and mappings between them than on distinctively model-theoretical constructions and techniques. It is therefore plausible to think that the tasks set by Suppes for a model-theoretic investigation of the mathematical models used in empirical science cannot be exhaustive. Such an impression is confirmed by a few notable applications of model theory to the theory of measurement, which have been largely neglected by the philosophical literature despite their importance: two of them are P.J. Cameron's classification of rational-valued scale types, obtained in Cameron (1989), and the construction of measurement values as model-theoretic types presented in Niederée (1992). Informally speaking, Cameron's result shows that, if one thinks of empirical variables as dense orderings that are not continua (isomorphic to the ordered reals), the number of distinct scales they can theoretically give rise to increases from three 
(for continua) to infinity. ${ }^{1}$ Niederée's results have shown, among other things, how one can identify measuring numbers with sets of experimental data, as well as the equivalence between certain mathematical assumptions (e.g. the Archimedean property) and properties of experimental procedures. These results shed light on important features of scientific models (especially measurement models) by modeltheoretic means, without pursuing any of the tasks recommended by Suppes, i.e., theory formulation or data analysis. The main objective of this paper is to extend along a further direction the same model-theoretic style of investigation, which recognises the value of Suppes' original proposal but transcends its limited scope. The model-theoretic machinery I shall rely upon comes from Nonstandard Analysis: it is briefly surveyed in Sect. 2 (more details are found in the "Appendix"). I shall apply Nonstandard Analysis to two models from utility theory in order to construct an alternative mathematical treatment for the economic setups they are supposed to describe. This will allow me to show that the fragments of economic theory based on these models are crucially sensitive to a choice of mathematical treatment, more precisely, a selection of set-theoretic parameters. What this suggests is that economic theory is, at an abstract level, significantly sensitive to the choice of mathematical resources employed in its articulation. The existence of distinct choices leads to bifurcations in the kind of result one may hope to obtain. In particular, if one wishes to uphold certain normative constraints or introduce certain formal approaches, it is sometimes mandatory to drop traditional mathematical environments based on the real numbers. These remarks will be illustrated in full detail in Sects. 4-7, after a brief semi-technical preliminary.

\section{Classical and Nonstandard Analysis}

A vast amount of work in mathematical social science (especially economics) relies on the availability of the objects of classical analysis in the semantic metatheory. For example, in consumer theory utilities are real numbers, bundles of goods are real-valued vectors and their totality is canonically a subset of some Euclidean space. In many interesting cases there is no particular empirical motivation to select certain specific analytical objects in model-building, either because they (e.g. the metric structure on a set of alternatives ranked by a preference relation) support abstract models without having any empirical interpretation or because, even when they represent some non-mathematical content, they enter a model also as carriers of properties that have no particular connection with this content (e.g. the topological separability of the real numbers representing utilities) and yet influence what can be established about the given model. Because of this, it is of interest to consider what happens if one replaces certain canonically employed analytical objects with alternative objects. In this paper, I consider the objects of Nonstandard Analysis, ${ }^{2}$

\footnotetext{
${ }^{1}$ Here scales are distinguished on the basis of the number of reference points, e.g. origins or units, that uniquely determine them.

2 As is well-known, Nonstandard Analysis was created by Robinson (1966). The most widely adopted approaches in this field are the superstructure approach (see e.g. Davis 1977) and the axiomatic approach based on Nelson's Internal Set Theory (Nelson 1986; Robert 1988, but see also, as a result of many
} 
which share a number of properties with classical objects but are, at the same time, significantly different. I shall focus on their application to two mathematical models from utility theory. In each case, I study the consequences of using certain extensions of classical numerical sets within a Nonstandard universe as codomains of functions that are canonically selected to be real-valued. While a standard mathematisation based on real-valued functions gives rise to negative results, a Nonstandard mathematisation replaces them by positive results (which may hold under stronger conditions than were sufficient to deduce the negative results by standard means). This divergence highlights the essential relativity (i.e., with respect to a selection of mathematical resources) of negative results in economic theory, since the remarks that hold for the utility models discussed in detail admit of a general reformulation. Such a reformulation will be presented in Sect. 7, after a full discussion of the main examples has taken place, in Sects. 4-6. It is appropriate to note at this point, by way of a concluding remark, that applications of Nonstandard Analysis are not new to the field mathematical economics (see for instance, Skala 1975; Fishburn and Lavalle 1991; Lehmann 2001). However, all those of which I am aware adopt a local point of view, i.e., they construct an ultraproduct of some real structure suitable to specific modelling purposes. Moreover, they are not concerned with showing how canonical and nonstandard resources affect in divergent ways the results of modelling. My approach, on the contrary, is global in the sense that it relies on a Nonstandard universe in which several results involving nonstandard models are simultaneously obtained (this point will be clarified in Sect. 3). Moreover, it is primarily concerned with showing how canonical and nonstandard resources affect the results of modelling.

\section{A Formal Preliminary}

The Nonstandard universe I shall make use of in the next sections can be constructed from a collection $S_{0}$ of Urelemente (informally speaking, non-sets) that contains a copy of the set of real numbers $\mathbb{R}$. One can use $S_{0}$ to generate a hierarchy of set-theoretical objects by means of the following inductive condition:

$$
S_{n+1}=S_{n} \cup \mathcal{P}\left(S_{n}\right),
$$

where $\mathcal{P}\left(S_{n}\right)$ is the powerset of $S_{n}$. The union $U$ of all the $S_{i}$ (with $i$ a natural number) is a very rich object. It clearly contains the real numbers and all of their subsets, but it also contains the Cartesian product $\mathbb{R}^{n}$, for any natural number $n$, and, as a consequence, every finitary relation on the real numbers, all functions of one or several real variables and so on. The object $U$ can then be described by means of a first-order language with identity $L$ which contains, apart from connectives and quantifiers, the symbol $\in$ for set-theoretical membership and a name for every entity

Footnote 2 continued

refinements, Hrbacek et al. 2014). Here I make use of a version of the superstructure approach, which is outlined in the "Appendix" and proceeds along the lines of Bell and Machover (1978). 
in $U$ (including all relations, functions, sets of relations or functions etc.). ${ }^{3}$ Thus, in particular, there are L-names for $\mathbb{N}$, the set of natural numbers, or $\mathbb{S}$, the set of all sequences of real numbers, both of which will be considered later. One may then use the compactness theorem of first-order logic to obtain an enlargement $U^{\prime}$ of the structure $U=\langle U, \in\rangle$ (how this can be done is outlined in the "Appendix"). What matters for present purposes is that an arbitrary enlargement will contain extended numerical sets $* \mathbb{N}$ and ${ }^{*} \mathbb{R}$ that are richer than their respective counterparts $\mathbb{N}, \mathbb{R}$, in the sense that the latter numerical sets can be embedded in their starred extensions and these include additional elements. The only additional numerical elements that will be extensively relied upon in what follows are the infinitely large numbers in ${ }^{*} \mathbb{N}$, i.e., the elements in this set that are greater than any $n \in \mathbb{N}$ (where 'greater than' is a binary relation on ${ }^{*} \mathbb{N}$ that agrees with the usual ordering on the standard numbers). The language $L$ introduced above will also play an important role, since it allows one to state sentences that may contain names for $\mathbb{N}$ or $\mathbb{R}$ as parameters: if these sentences are true in the standard universe $U$, then they are true in its enlargement, with respect to $* \mathbb{N}$ and $* \mathbb{R}$. In Sect. 6 , this will make it possible to 'extend' properties of arbitrary, standard numbers to infinitely large numbers in particular. This preliminary is sufficient to introduce the models alluded to in the previous section.

\section{Ranking Information States}

My first example is taken from choice theory. A typical aim in this setting is to show that the choice-behaviour of an agent can be represented by a utility function. Formally, one introduces a space $A$ of alternatives over which the agent is supposed to express or reveal her preferences by way of binary comparisons. Thus, a preference is understood to be a binary relation $P$ on $A$, in particular a complete preorder, i.e., a relation that is transitive and complete. The aim is then to show the existence of a function $u: A \rightarrow \mathbb{R}$, from $A$ into the set of real numbers $\mathbb{R}$ such that, for any $x, y \in A$ :

$$
x P y \text { iff } u(x) \leq u(y) .
$$

It is not unusual to encounter in the literature models where $P$ is defined on an uncountably large set. A fairly recent collection of models based on this setting, the simplest of which I am going to discuss, is found in Dubra and Echenique (2001). Given the specific purpose of this paper, I shall accept a few otherwise problematic assumptions characteristic of these models. Such assumptions notably include the postulation that choice outcomes give rise to a complete preorder (so that, in particular, any two distinct alternatives can be ranked and indifference is transitive) and the presupposition that uncountably large spaces of alternatives provide an acceptable idealised framework. Questionable as these assumptions may be, my

\footnotetext{
${ }^{3}$ It proves convenient to have in the language also a two-place $\langle$,$\rangle term-forming operator that designates$ ordered pairs. This term does not need to be assumed, since it could be introduced using the language L. I have assumed its availability in Sect. 6 .
} 
main aim is to retain them in order to show that, once they have been deployed, the highly idealised character of the resulting models makes them amenable to distinct mathematical treatments that, in turn, give rise to vastly different results. In brief, substantive appeals to mathematical idealisation give rise to divergent modelling trajectories. Although I shall focus on the most basic model studied by Dubra end Echenique, it is worth noting that my remarks about it also apply, essentially unchanged, to more sophisticated variants presented in the same paper. The model in question involves an uncountably large set $\Omega$ of possible 'states of nature' endowed with the family of its partitions. Each partition divides the whole $\Omega$ into disjoint subsets and a partition $\mathscr{P}$ is finer than a partition $\mathscr{Q}$ if every element of $\mathscr{P}$ is included in some element of $\mathscr{Q}$ (if strictly included, then $\mathscr{P}$ is strictly finer than $\mathscr{Z}$ ). This formal model is meant to describe in mathematical terms the informational states of a decision-maker: one interprets an arbitrary partition of $\Omega$ as resulting from an equivalence relation of informational indifference. For an agent in possession of $\mathscr{Q}$, any two possible states of nature within an element of $\mathscr{Z}$ carry the same amount of information and are therefore indistinguishable from this point of view. It is also assumed that any decision-maker would find the transition from 2 to a strict refinement $\mathscr{P}$ desirable, since it leads from a less informative to a more informative state. With this description of the model in place, taking $\Pi(\Omega)$ to be the set of all partitions of $\Omega$, Dubra and Echenique consider the complete preorders on $\Pi(\Omega)$ that rank any partition strictly below any strict refinement (a condition they call monotonicity). According to them, any rational decision-maker must rank $\Pi(\Omega)$ on one such preorder. It can however be shown that none of them is representable by a real-valued utility function. Dubra and Echenique draw the following conclusion:

Our result is important because it shows that utility theory is not likely to be a useful tool in the analysis of the value of information. This finding should be contrasted with the existing literature on the value of information, where utility representations are used. The use of a utility implies that preferences are not monotone (Dubra and Echenique 2001: 1).

The point of the above quotation is that the existence of a utility representation is incompatible with the assumption that an agent should prefer more information to less. This remark is certainly correct, but it hides the following dilemma: is the problem inherent in utility theory as a formal approach to the study of idealised rankings or is it an effect of restricting attention to real-valued utility functions and, thus, of certain properties of $\mathbb{R}$ ? This dilemma refines the formulation of the problem highlighted by Dubra and Echenique because it does not presuppose that the codomain of a utility function should be $\mathbb{R}$. No particular feature of the space of informational states suggests that such a codomain should be selected. It is therefore meaningful to look for alternative numerical codomains, on which utility functions may exist. In other words, it is reasonable to conjecture that a lack of fit exists not between utility functions and spaces of information states, but between these spaces and the ordered reals. An application of Nonstandard Analysis shows that bounded utility functions on $* \mathbb{N}$ exhibit a much better fit: 
Theorem 4.1 Every complete preorder has an uncountable family of bounded utility functions on $* \mathbb{N}$.

The proof that a bounded utility function on ${ }^{*} \mathbb{N}$ exists is included in the "Appendix". Since any strictly increasing, monotonic transformation of a utility function is a utility function, multiplying the values of one by the infinitely large number $H \in{ }^{*} \mathbb{N}$ yields a utility functions. Since there are uncountably many such numbers (see "Appendix"), there are uncountably many utility functions. ${ }^{4}$ In the light of Theorem 4.1, the suggestion that utility theory is not likely to be a useful tool in the analysis of the value of information may be successfully resisted. The applicability of utility theory as a uniform approach to the representation of idealised preferences can be rescued, provided that one does not restrict attention to real-valued utilities, which may be incompatible with the structure of certain preorders. Note that, since ${ }^{*} \mathbb{N}$ is compatible with all of them, it is a numerical set and shares with $\mathbb{N}$ every formal property statable in $L$, a language powerful enough to express the whole of classical mathematics, there does not seem to be any natural objection to the transition from the codomain $\mathbb{R}$ to $* \mathbb{N}$. The latter transition may also be regarded as the shift from one mathematical treatment, in which a particular selection of semantic resources has been made, to an alternative treatment, based on an alternative selection. The striking fact is that, on the first treatment, a certain problem (representing monotone rankings of information states) admits of no solution, whereas, on the alternative treatment, it admits of uncountably many. At a certain level of mathematical idealisation, the selection of semantic resources makes a huge difference to the implications of a model. This phenomenon is also responsible for the intimate connection between semantic resources and normative assumptions that some models display, as will be seen in the next two sections.

\section{Discounting the Future}

A simple and interesting illustration of the effects of alternative mathematical treatments on normative constraints, which belongs, as the example from Sect. 4, to utility theory, is a particular aggregation problem that arises in decision-making scenarios involving policy choices with repercussions on the distant future. Decisions concerning e.g. radioactive waste disposal, natural resource depletion, levels of pollution that have an impact on biodiversity fall under this category. Since future generations are affected by these decisions, it is plausible to think of the relevant alternatives as sequences of utility indices, each of which expresses the overall welfare level of one generation or one time-period. This approach has been pursued since at least Diamond (1965), who studied infinite utility streams over

\footnotetext{
${ }^{4}$ The existence of a utility function on a suitable ultraproduct of the reals is proved in Skala (1975: 44) and Narens (1985: 258-259). Theorem 4.1 shows that one does not in general need to consider the reals, since a suitable extension of $\mathbb{N}$ suffices. Moreover, Narens and Skala are primarily interested in the existence of at least one utility function, and do not emphasise that, in fact, a large family of them can be obtained. It is worth pointing out that Theorem 4.1 goes through for $A$ of arbitrary, infinite cardinality, although any given cardinality will call for an enlargement that satisfies suitable saturation properties.
} 
certain (metrizable) topological spaces, and has received renewed attention over the last decade. The best way to understand the mathematical setup at play here is to consider the case of finite utility streams first. Suppose that numerical utility indices are attached to successive periods of time, e.g. generations, and only a finite number of future periods is taken into account. Then the alternatives over which a choice is to be made may be described by finite, ordered sequences of real numbers, i.e., finite utility streams. Thus, for $n$ periods, a utility stream is a vector $s=\langle s(1), \ldots, s(n)\rangle$, with $s(i), i=1, \ldots, n$ a natural number. In order to compare distinct utility streams, one may think of assigning to each one of them a numerical index: then a stream will be preferred to another when its aggregate index is not smaller than the index assigned to the other stream. An easy, conventional way of aggregating a stream $s$ consists in defining its aggregate index to be the sum of its components. When this is done, the totality of finite $n$-ary streams is mapped into the ordered natural numbers. The mapping $u$ thus obtained allows a direct comparison between streams which satisfies two intuitively desirable properties. In order to state them, it is convenient to define two binary relations between streams, symbolised by $\succ$ and $p$ respectively. The first relation holds when $s(i) \geq t(i)$ for $i=1, \ldots, n$, with strict inequality for some $i$; the second does when $t$ is obtained from $s$ by a finite permutation $^{5}$ of its components. The two properties in question are:

- $\quad$ Pareto (P): $s \succ t$ implies $u(s)>u(t)$.

- Anonymity (A): $p(s, t)$ implies $u(s)=u(t)$.

Condition (P) says that the comparison of aggregated streams is sensitive to improvements in a single time-period, whilst condition (A) says that the utility indices of the present and of future time-periods are equally important. It is thus trivial to establish Paretian and Anonymous aggregations for finite utility streams. An interesting problem arises when one seeks to use utility streams to describe an indefinitely remote future: perhaps the most obvious way of doing so is to deal with infinite utility streams, i.e., infinite sequences of utility indices. Then, even if one assumes that only finitely many distinct utility indices, but at least two of them, may occur along a stream, the space of alternatives is uncountably large, exactly as in the model from Sect. 4. This analogy may be brought further, since it can also be proved for this uncountable object that no real-valued function defined on it and satisfying (P) and (A) exists. Basu and Mitra (2003) proves this result for infinite, binary streams. Crespo et al. (2009) derives the same theorem from (A) and a condition strictly weaker than $(\mathrm{P}){ }^{6}$ In view of the previous section, one might expect that such a negative result could be bypassed by a suitable choice of alternative mathematical resources. I shall consider two alternative ways of achieving this goal. The first is straightforward but may be regarded as unsatisfactory, for reasons to be clarified in a moment.

\footnotetext{
${ }^{5}$ Here permutations cannot but be finite. I emphasise finiteness because it will appear also in the discussion of infinite utility streams to follow.

${ }^{6}$ In the present notation, this condition states that $s \succ t$ implies $u(s)>u(t)$ if strict inequality holds for infinitely many components of $s, t$ (see Crespo et al. 2009, p. 52).
} 
Lemma 5.1 Let $\mathbb{S}$ be the set of real-valued, infinite utility streams. There is an aggregation $u$ from $\mathbb{S}$ to an initial segment of $* \mathbb{N}$ that satisfies $(P)$ and $(A)$.

Proof Following Svensson (1980), define the binary relation $P$ on $\mathbb{S}$ by the following condition on any $s, t \in \mathbb{S}$ :

$$
s P t \text { iff for some finite permutation } p \text { of } \mathbb{N}, p(s) \geq t
$$

where the relation ' $\geq$ ' is component-wise inequality. It can be verified that $P$ is a preorder that satisfies (P) and (A). By an application of the Axiom of Choice known as Szpilrajn lemma, $P$ can be extended to a complete preorder, which is representable by some function $u$ on an initial segment of $* \mathbb{N}$ by Theorem 4.1. The representation $u$ is also an aggregation on $\mathbb{S}$ that satisfies (P) and (A).

A fortiori, Lemma 5.1 ensures the existence of an aggregation $u$ when infinite utility streams take values in $\mathbb{N}$. Nevertheless, this result does not establish any continuity between the finite and the infinite case: in particular, it does not decide whether it is possible to aggregate utilities by summation in both cases. The latter possibility may be viewed as a desirable requirement because homogeneity in aggregation could be taken as evidence that infinite utility streams are a good generalisation of finite utility streams. It turns out that, for utility streams that take only finitely many values, - a plausible restriction-this type of homogeneity can be established only if one gives up (A), in particular by discounting the future. Such a move is not unfamiliar to economists, since e.g. it is often adopted to solve optimal control problems over an infinite time horizon. In the present context a discounting approach takes the following form:

Remark Let $S_{k}$ be the set of all infinite utility streams generated by a finite set of real numbers $\left\{r_{1}, \ldots, r_{k}\right\}$. There are uncountably many discounted utility representations of $\mathbb{S}$ that satisfy $(\mathrm{P})$.

Proof Set $r=\max \left\{r_{1}, \ldots, r_{k}\right\}$ and fix an arbitrary, positive real $a>1$. The constant sequence $\langle r, r, r, \ldots\rangle$ bounds above every other sequence in $\mathbb{S}_{k}$ componentwise. Now consider the discounting factor $a^{-n}$ (which equals $a^{0}=1$ on the first component of a utility stream, $a^{-1}$ on the second, and $a^{-n}$ on the $n$ th. For any $s \in \mathbb{S}_{k}$ with $n$th component $s_{n}$, consider the series:

$$
u(s)=\sum_{i=0}^{\infty} s_{n} a^{n} .
$$

This series is bounded above by the series obtained by setting $s_{n}=r$, for every $n$. Since the latter series has a sum, namely $r e^{a}$, the former does. As a result $u$ is a discounted aggregation for $\mathbb{S}_{k}$ : because there are uncountably many choices for $a$, there are uncountably many such aggregations.

In the light of the last remarks alone, one may conjecture that the only way to obtain a 'natural' aggregation (i.e., by summation) for utility streams is to impose a discount rate on the future. If this conjecture were true, then, theoretically, there would be no way of implementing an ethically desirable condition like (A) by way 
of an aggregation. ${ }^{7}$ It would look as though one were forced to drop (A) and adopt the normatively problematic assumption that utility decays at a certain rate in time, if one wished to compare alternatives by means of aggregations. This standpoint is, however, not reasonable, since it would lead to theoretical scenarios in which e.g. the faster-paced consumption of an exhaustible resource or a more intensive exploitation of natural resources was deemed preferable to an alternative policy, independently of its environmental effects. One might advocate such an ethically unacceptable perspective only by suggesting that more utility is to be extracted from a certain resource in the present or near future than it could be in the remote future. ${ }^{8}$ Abstractly speaking, such an argument has any force only insofar as one takes the semantic components of the model that licenses it to be fixed. When this constraint is abandoned, it becomes meaningful to search for a mathematical treatment that satisfies (A) and rules out discounting future utilities. There is no particular reason to take the set-theoretical parameters of the aggregation problem as fixed and then test the satisfiability of (A) under these parameters. In fact, there are ethical reasons to subordinate the choice of mathematical resources to the satisfiability of a normative constraint like (A). In other words, one may wish to detect the mathematical resources that conflict with (A) in order to replace them with others that do not. In fact, discount-free aggregations that add utilities and satisfy both $(\mathrm{P})$ and (A) can be obtained if one chooses the codomain of the aggregation to be $* \mathbb{R}$ instead of $\mathbb{R}$ (or ${ }^{*} \mathbb{N}$ instead of $\mathbb{N}$ ). The applicability of Nonstandard Analysis to this problem is adumbrated (but not fully articulated) in Lauwers (2010) ${ }^{9}$ and has been briefly illustrated in Pivato (2014) but none of these authors has emphasised it as an instance of the effects of alternative mathematical treatments. Furthermore, none of them points out that one can easily obtain an uncountable family of aggregations satisfying (A) and (P). This is proved in the next section.

\section{Discount-Free Aggregations}

Within the Nonstandard universe quickly described in Sect. 3 (see also the "Appendix"), any real-valued, infinite utility stream $s$ has a * $\mathbb{R}$-valued extension ${ }^{*} s$ : since $s(n)=r$ is a true L-sentence about $s$, it follows that, for every $n \in \mathbb{N}, s(n)={ }^{*} s(n)$. The difference between these two sequences is that ${ }^{*} s$ also has infinitely large arguments, at which it takes uniquely determined values. An additive, discount-free representation of utility streams may be obtained by truncating ${ }^{*} s$ at an arbitrary, infinitely large argument. Since, intuitively, the truncation of ${ }^{*} s$ at any argument behaves like a finite subsequence, its values can be * summed, in the sense that one applies to them a function which is the nonstandard extension of a finite summation, also known as a *finite, or hyperfinite, summation.

\footnotetext{
7 On the other hand, it would be possible to determine an intrinsic ranking of $\mathbb{S}$ that satisfies (P) and (A). This possibility is actually exploited in the proof of Lemma 5.1.

8 The locus classicus for a critique of discounting on ethical grounds is Ramsey (1928). A more recent critique of discounting in the context of environmental economics may be found in Weitzman (1998).

9 Lauwers shows how one may directly order utility streams by means of an ultrafilter-based argument which would determine the same aggregate ranking on a corresponding ultrapower of the reals.
} 
Taking such a summation preserves all information carried by $s$ (since $s$ and ${ }^{*} s$ agree at every finite argument) in a format that can be handled much like an ordinary, finite summation. This allows a straightforward generalisation of the treatment of finite utility streams described in Sect. 4. In particular both (P) and (A) are satisfied by *finite summations because of the following two lemmas ( $\mathbb{S}$ below is the set of real-valued, infinite utility streams):

Lemma 6.1 If $s, t \in \mathbb{S}$ and $H$ is an infinitely large number, then:

$$
p(s, t) \text { implies } \sum_{i=0}^{H}{ }^{*} s(i)=\sum_{i=0}^{H}{ }^{*} t(i) \text {. }
$$

Lemma 6.2 If $s, t \in \mathbb{S}$ and $H$ is an infinitely large number, then:

$$
s \succ \text { timplies } \sum_{i=0}^{H}{ }^{*} s(i)>\sum_{i=0}^{H} * t(i) .
$$

Lemmas 6.1 and 6.2 in turn imply:

Theorem 6.3 Let $\mathbb{S}$ be the set of all infinite, real-valued utility streams. There is an uncountable family of $* \mathbb{R}$-valued aggregations for $\mathbb{S}$ satisfying both $(P)$ and (A). ${ }^{10}$

The last theorem shows that normative constraints may be deeply connected to a choice of mathematical resources. Under a canonical choice (real-valued aggregations) there is no way of meeting constraint (A). An alternative choice gives rise to uncountably many ways of meeting it. The latter choice is not only preferable on ethical grounds, i.e., because it gives rise to a theoretical model that does not presuppose the viability of unacceptable policies, but may even be supported on technical grounds. This is clarified by two representative quotations from economists working on the problem of intertemporal choice and finding it problematic to work with the reals. The first quotation comes from Koopmans:

[...] there is not enough room in the set of real numbers to accommodate and label numerically all the different satisfaction levels that may occur in relation to consumption programs for an infinite future (Koopmans 1960: 288)

The very same issue is raised by Basu and Mitra when they point out that:

\footnotetext{
${ }^{10}$ In fact, these aggregations do not only satisfy (P) and (A) but also a further interesting condition that does not follow from them. This is called 'Hammond equity for the future' $(\mathrm{H})$ and has been introduced in Asheim and Tungodden (2005): formally, it states that, if $s(i)>t(i)$ for $i \geq 2$ and $s(1)<t(1)$, then $u(s)>u(t)$. The intuitive motivation for $(\mathrm{H})$ is the idea that a relative loss in the present is acceptable if it is to be followed by a relative gain for all future generations. The plausibility of this condition depends, among other things, on the rather optimistic assumption that correct estimations of future utility levels can be made for an arbitrary distant future.
} 
The proof of our result, roughly speaking, involves showing that in trying to represent any social welfare function respecting equity and the Pareto principle [these are (A) and (P) above], one "runs out of real numbers". (Basu and Mitra 2003: 1558)

These quotations seem to reveal a recognition of the fact that there is an inherent problem with reconciling the structure of the real numbers with that of certain formal preferences. This may be read as a statement against the use of real numbers under these circumstances. The fact that one 'runs out of real numbers' is not to be seen as an obstacle to tackling an aggregation problem, but rather as a call for methods that can tackle them.

\section{Impossibility as Unsatisfiability}

The results from Dubra and Echenique (2001), Basu and Mitra (2003) and Crespo et al. (2009), which have been discussed above, are instances of what are often referred to in the economic literature as impossibility theorems. Roughly speaking, an impossibility theorem states that certain conditions cannot be simultaneously met. As the previous sections have shown, it would be incorrect to take them to assert the absolute inconsistency of a set of conditions. This is because such inconsistency holds only when certain parameters occurring within the relevant statements have been fixed, but may disappear under an alternative selection of parameters. In order to clarify this remark, it is helpful to articulate it with the aid of some elementary logical notions. Virtually every impossibility theorem obtained in the economic literature relies on a piece of classical mathematics and, as such, it determines a L-formula with one free variable that is unsatisfiable in $U$. Thus, impossibility is better qualified as unsatisfiability and an impossibility theorem is better characterised as a metatheoretical statement to the effect that a certain formula is false in $U$ under every assignment of a set-theoretical entity to its free variable. To see this more explicitly, consider the impossibility result obtained in Basu and Mitra (2003) and note that $L$ has a name for the set $\mathbb{S}$ of all real-valued utility streams and can express the binary relations and $\succ$ and $p$ respectively. With an abuse of notation (using e.g. $\mathbb{S}$ also as a name in the language for the set $\mathbb{S}$ ), one can write the following (abbreviated) L-formula:

$$
\begin{aligned}
& x \in \mathcal{P}(\mathbb{S} \times \mathbb{R}) \wedge(\forall u \in \mathbb{S})(\exists v \in \mathbb{R})(\langle u, v\rangle \in x) \wedge(\forall u \in \mathbb{S})(\forall v \in \mathbb{R})(\forall z \in \mathbb{R})((\langle u, v\rangle \in \\
& x \wedge\langle u, z\rangle \in x) \rightarrow v=z) \wedge(\forall u \in \mathbb{S})(\forall v \in \mathbb{S})(\forall r \in \mathbb{R})(\forall s \in \mathbb{R})((u \succ v \wedge\langle u, r\rangle \in \\
& x \wedge\langle v, s\rangle \in x) \rightarrow r>s) \wedge(\forall u \in \mathbb{S})(\forall v \in \mathbb{S})(\forall r \in \mathbb{R})(\forall s \in \mathbb{R})((p(u, v) \wedge\langle u, r\rangle \in \\
& x \wedge\langle v, s\rangle \in x) \rightarrow r=s) .
\end{aligned}
$$

Call the last formula $\theta(x)$ : if $\theta(x)$ were satisfiable, then there would be a total, single-valued correspondence from $\mathbb{S}$ to $\mathbb{R}$ (a utility function) satisfying $(\mathrm{P})$ and $(\mathrm{A})$ (this is what the formula says). What Basu and Mitra proved can be reduced to the statement that $\theta(x)$ is unsatisfiable in $U$. It does not follow that the same formula cannot be satisfied elsewhere. If one interprets the symbols $\mathbb{R}$ and $\mathcal{P}(\mathbb{S} \times \mathbb{R})$ 
occurring in $\theta(x)$ respectively on the entities $* \mathbb{R}$ and $\mathcal{P}^{*}(\mathbb{S} \times \mathbb{R})$ (i.e., the powerset of the entity $*(\mathbb{S} \times \mathbb{R})$ ), then Theorem 6.3 shows that $\theta(x)$ is satisfiable in the Nonstandard universe ${ }^{*} U \theta(x)$. Alternatively, one might have used a variant of in which the parameters $\mathbb{R}, \mathbb{S} \times \mathbb{R}$ had been replaced by suitable nonstandard parameters (attached to an interpretation on the Nonstandard universe). In this case, a change in mathematical treatment would have been highlighted by a change of parameters, which is attached to a change of semantic resources, given the interpretation of the parameters. The availability of distinct mathematical treatments amounts, in this simple scenario, to the possibility of selecting parameters attached to distinct semantic environments. When one deals with highly idealised models, as is frequently the case in mathematical economics, no particular selection is forced by the empirical elements of the model. As a result, the option of removing an impossibility theorem by a suitable choice of mathematical treatment is in general open. This means that impossibility theorems should be regarded as statements of relative impossibilities constrained by a selection of mathematical parameters. Relative impossibilities can thus be removed without changing the linguistic formulation of the conditions that imply them under a specific choice of parameters. The example of $\theta(x)$ just discussed offers an illustration of this point and suggests a novel strategy to deal with relative impossibilities. Whereas, traditionally, negative results are circumvented by replacing some of their assumptions by weaker ones that no longer imply them, the previous analysis shows that the same, or even stronger assumptions may be retained, provided that a suitable change of parameters is effected. Recall, for instance, that, in the case of infinite utility streams, Theorem 6.3 removes the impossibility result proved in Crespo et al. (2009) under stronger conditions, namely (P) and (A), than those assumed in that paper (see footnote 6 ). The same theorem also removes the impossibility result proved in Basu and Mitra (2003) under the strictly stronger set of conditions (P), (A) and (H) (see footnote 10). These remarks are entirely general, since one may write a formula $\theta(x)$ with one free-variable for any known impossibility theorem. To replace $\theta(x)$ with a different formula $\theta^{\prime}(x)$ for which no impossibility can be proved is to circumvent an impossibility. To retain $\theta(x)$ and change the interpretation of its parameters is, on the other hand, to remove it. Even though the latter strategy may be in principle practicable, it is not guaranteed that it should always be fruitful. The examples discussed in this paper show however that there are interesting cases in which its fruitfulness is sufficiently evident.

\section{Concluding Remarks}

It is easy to think of mathematics as a system of resources whose main effect on modelling is to constrain the setup to which they are applied, by deploying forms of reasoning that conclusively establish results and exclude alternatives. This picture captures one of the advantages of any mathematical treatment, namely control over the object of investigation, but neglects another, distinct, character of mathematical modelling, namely its open-endedness. As the examples discussed in the previous 
sections have shown, mathematical resources offer the means to construct alternative treatments, which may give rise to different modelling trajectories. Mathematics is thus not only to be seen an instrument that stringently fixes outcomes by way of proofs, but also as the matrix of a plurality of formal strategies. As the previous sections have shown, the latter characteristic of mathematics has a non-negligible impact on modelling practices, in view of its close connection with the viability of certain formal approaches or the satisfiability of certain normative constraints.

Open Access This article is distributed under the terms of the Creative Commons Attribution 4.0 International License (http://creativecommons.org/licenses/by/4.0/), which permits unrestricted use, distribution, and reproduction in any medium, provided you give appropriate credit to the original author(s) and the source, provide a link to the Creative Commons license, and indicate if changes were made.

\section{Appendix}

\section{Construction of a Nonstandard Universe}

In order to construct $U^{\prime}$, consider the family $C$ of all sets of $U$-concurrent $\mathrm{L}$-formulae: these are sets of formulae with one free variable that are finitely satisfiable in $U$. For instance, consider the countable set of all formulae of the form $x \in \mathbb{N} \wedge x \neq n$, with $n$ a constant denoting a natural number: since there are infinitely many natural numbers, each finite subset of this set is satisfiable for some assignment of a sufficiently large natural number to the variable $x$. It can be proved using the compactness theorem (see Bell and Machover 1978: 534) that, turning $C$ into a family of sets of sentences by means of new constant symbols, ${ }^{11}$ the resulting family $C^{\prime}$ determines a model $U^{\prime}$ of $\cup C^{\prime}$, such that $U$ is an elementary substructure of $U^{\prime}$. This is to say that there is an elementary embedding * $U \rightarrow U^{\prime}$, which in particular sends each $S_{i}$ (defined in Sect. 3 above) into ${ }^{*} S_{i}$. The union $\bigcup_{i \in \mathbb{N}}{ }^{*} S_{i}$ is the nonstandard universe ${ }^{*} U$, whose entities are called internal entities. Note that the nonstandard universe is a sufficiently rich object: for instance, since both $\mathbb{N}$ and $\mathbb{R}$ are elements of $S_{1}$, it follows that $* \mathbb{N}$ and ${ }^{*} \mathbb{N}$ are internal entities in ${ }^{*} U$. By the same clue, since every set of ordered pairs of reals is an element of $S_{3}$, the relation ${ }^{*}<$, as well as any function of one real-variable, has its counterpart in ${ }^{*} S_{3}$. It is convenient to introduce a language ${ }^{*} \mathrm{~L}$ that has the same resources as $\mathrm{L}$ but, instead of names for the entities in $U$, has names for the internal entities in ${ }^{*} U$. The ${ }^{*}$-transform of a L-sentence is obtained by replacing its constants with the corresponding starred symbols. Since $U$ is an elementary substructure of $U^{\prime}$, it follows that an L-sentence is true in $U$ iff its *-transform is true in $U^{\prime}$. The crucial feature of $U^{\prime}$ that makes it an interesting superstructure of $U$ and will be exploited below is that every set of $\mathrm{L}$-sentences concurrent in $U$ determines a set of ${ }^{*} \mathrm{~L}$-sentences that are simultaneously satisfied by some entity in the domain of $U^{\prime}$ (which we are usually able to locate as an internal entity in ${ }^{*} U$ ). For instance, consider again the set of $\mathrm{L}$-sentences of the form $x \in N \wedge x \neq n$ : the corresponding set of * $\mathrm{L}$-sentences is the

\footnotetext{
${ }^{11}$ Each set of $U$-concurrent formulae is turned into a set of sentences by replacing the free variable in each of these formulae by the same, new (i.e., not in L) constant symbol.
} 
denumerable set whose elements have the form $x \in{ }^{*} \mathbb{N} \wedge x \neq{ }^{*} n$. Since we may identify ${ }^{*} n$ and $n$, it follows that ${ }^{*} \mathbb{N}$ contains some number $H$ that differs from every natural number ${ }^{*} n=n$. Note that $H$ cannot be smaller than any ${ }^{*} n$, since every natural number smaller than $n$ must be one of $0,1, \ldots, n-1$ (this can be stated as a L-sentence true in $U$, which therefore has a (true) ${ }^{*}$-transform). Thus, since the natural numbers are linearly ordered (again, a true L-sentence with a ${ }^{*}$-transform), $H$ is an infinitely large number. As a consequence, the initial segment $1, \ldots, H$ of $* \mathbb{N}$ is an infinite set and it can be proved that it is uncountable (see e.g. Goldblatt 1998: 197). In general, any infinite set in $U$ contains an enlargement with additional elements in ${ }^{*} U$ : for numerical sets, this form of enrichment implies the availability of additional numbers.

\section{Proof of Theorem 4.1}

Theorem 4.1 is a corollary of the following result:

Theorem 8.1 Let $A \subset S$ be a nonempty set and $P$ be a complete preorder on $A$. Then there is a function $u^{+}: A \rightarrow{ }^{*} \mathbb{N}$ such that, for any $x, y \in A: x P$ iff $u^{+}(x) \leq u^{+}(y)$.

Proof Let $\mathcal{P}_{F}(A)$ be the set of the finite subsets of $A$. Any $s \in \mathcal{P}_{F}(A)$, being finite, can be represented on $\mathbb{N}$ by some suitable function $u$. Now consider the binary relation $R$ between subsets of $A$ and $\mathbb{N}$-valued functions defined by the following condition: $s R u$ iff $s \in \mathcal{P}_{F}(A)$ and $u$ is a $\mathbb{N}$-valued function (e.g. an element of the powerset $\left.\mathcal{P}_{F}(A \times \mathbb{N})\right)$ on $s$ such that, for any $x, y \in s: x P$ iff $u(x) \leq u(y)$. The relation $R$ is concurrent because, if $s_{i} R u_{i}(i=1, \ldots, n)$, then the union of the $s_{i}$ is a finite set with a $\mathbb{N}$-valued representation, which can be restricted to yield a representation for each of the $s_{i}$. The concurrence of $R$ implies the existence of a $* \mathbb{N}$ valued, internal function $u^{+}$such that, for every $s$ in the domain of $R,{ }^{*} s^{*} R u^{+}$. Now consider any $x, y \in A$ : since ${ }^{*}\{x, y\}$ belongs to the domain of ${ }^{*} R$, we have ${ }^{*}\{x, y\}^{*} R u^{+}$and $x P y$ iff $u^{+}(x) \leq u^{+}(y)$.

Proof of Theorem 4.1 Since any finite subset of $A$ has a representation on an initial segment of $\mathbb{N}$, one can adapt the proof of Theorem 4.1 to establish the existence of a utility representation on an initial segment of $* \mathbb{N}$.

\section{Proofs of the Results from Section 6}

Proof of Lemma 6.1 Since $p(s, t)$ holds, only finitely many elements of $s$ need to be reshuffled in order to obtain $t$. As a consequence, there is $n \in \mathbb{N}$ for which the Lsentence $\phi$ below holds:

$$
(\forall x \in \mathbb{N})\left(x>n \rightarrow \sum_{i=0}^{n} s(i)=\sum_{i=0}^{n} s(i)\right) .
$$

The ${ }^{*}$-transform of $\phi$, call it ${ }^{*} \phi$, is true in ${ }^{*} U$. Since any infinitely large number $H$ is greater than the finite number $n$, an instantiation of the universal quantifier for $H$ in $* \phi$ yields the result. 
Proof of Lemma 6.2 Substitute ' $>$ ' to ' $=$ ' in the L-sentence from Lemma 6.1: the same argument applies.

Proof of Theorem 6.3 Fix an arbitrary, infinitely large number $H$. The function $u: \mathbb{S} \rightarrow{ }^{*} \mathbb{R}$, defined by the following condition:

$$
u(s)=\sum_{i=0}^{H} * s(i)
$$

for any $s \in \mathbb{S}$, satisfies (P) and (A) by Lemmas 6.1 and 6.2. Since there are uncountably many, infinitely large numbers in ${ }^{*} \mathbb{N}$, there are uncountably many ways of defining $u$.

\section{References}

Asheim, G. B., \& Tungodden, B. (2005). A new equity condition for infinite utility streams and the possibility of being Paretian, Memorandum 08. Department of Economics, Oslo University.

Basu, K., \& Mitra, T. (2003). Aggregating infinite utility streams with intergenerational equity. Econometrica, 71, 1557-1563.

Bell, J. L., \& Machover, M. (1978). A course in mathematical logic. Amsterdam: North Holland.

Cameron, P. J. (1989). Groups of order-automorphisms of the rational numbers with prescribed scale type. Journal of Mathematical Psychology, 33, 163-171.

Crespo, J. A., Nuñez, C., \& Rincón-Zapatero, J. P. (2009). On the impossibility of representing infinite utility streams. Economic Theory, 40, 47-56.

Da Costa, N. C. A., \& Chuaqui, R. (1988). On Suppes' set theoretical predicates. Erkenntnis, 29, $95-112$.

Da Costa, N. C. A., \& French, S. (2003). Science and partial truth. Oxford: Oxford University Press.

Davis, M. (1977). Applied nonstandard analysis. New York: Wiley.

Diamond, P. A. (1965). The evaluation of infinite utility streams. Econometrica, 33, 170-177.

Dubra, J., \& Echenique, F. (2001). Monotone preferences over information. Topics in Theoretical Economics, 1, 1-16.

Fishburn, P. C., \& Lavalle, I. H. (1991). Nonstandard nontransitive utility on mixture sets. Mathematical Social Sciences, 21, 233-244.

Goldblatt, R. (1998). Lectures on the hyperreals. New York: Springer.

Hrbacek, K., Lessmann, O., \& O’Donovan, R. (2014). Analysis with ultrasmall numbers. New York: Academic Press.

Koopmans, T. C. (1960). Stationary ordinal utility and impatience. Econometrica, 28, 287-309.

Lauwers, L. (2010). Ordering infinite utility streams comes at the cost of a non-Ramsey set. Journal of Mathematical Economics, 46, 32-37.

Lehmann, D. (2001). Expected qualitative utility maximization. Games and Economic Behaviour, 35, 54-79.

Morgan, M., \& Morrison, M. (1999). Models as mediators. Cambridge: Cambridge University Press.

Narens, L. (1985). Abstract measurement theory. Cambridge, MA: MIT Press.

Nelson, E. (1986). Radically elementary probability theory. Princeton: Princeton University Press.

Niederée, R. (1992). What do numbers measure? A new approach to fundamental measurement. Mathematical Social Sciences, 24, 237-276.

Pivato, M. (2014). Additive representation of separable preferences over infinite products. Theory and Decision, 77, 31-83.

Ramsey, F. P. (1928). A mathematical theory of savings. The Economic Journal, 38, 543-559.

Robert, A. M. (1988). Nonstandard analysis. New York: Wiley.

Robinson, A. (1966). Nonstandard analysis. Amsterdam: North Holland.

Skala, H. (1975). Non-Archimedean utility theory. Dordrecht: Reidel.

Suppes, P. (1960). A comparison of the meaning and use of models in mathematics and the empirical sciences. Synthese, 12, 287-300. 
Svensson, L.-G. (1980). Equity among generations. Econometrica, 48, 1251-1256.

van den Bogaard, A. (1999). Past measurements and future prediction. In M. Morgan \& M. Morrison (Eds.), Models as mediators (pp. 282-325). Cambridge: Cambridge University Press.

Weitzman, M. L. (1998). Why the far-distant future should be discounted at its lowest possible rate. Journal of Environmental Economics and Management, 36, 201-208. 